\title{
Aoristo)))))
}

International Journal of Phenomenology, Hermeneutics and Metaphysics

\section{La nozione fenomenologica di dato}

The phenomenological notion of data

Prof.essa. Dr.essa. Roberta Lanfredini

Università degli studo do Firenze'

\section{RIASSUNTO}

In questo saggio sosterrò la tesi stando alla quale la prospettiva di Husserl va nella direzione di un empirismo estremamente sofisticato perché nello stesso tempo radicale ma non riduzionistico. Una forma di empirismo, cioè, che pur mirando a una revisione consistente dell'empirismo classico, non si discosta mai dal suo intento generale. Una delle obiezioni principali che possono essere rivolte a questa lettura è che l'utilizzo della nozione di a priori, più in particolare della nozione di a priori materiale, risulta essere sia storicamente sia teoreticamente contraria a una prospettiva di stampo empirista. Questa obiezione può essere in larga parte ridimensionata una volta compreso come le espressioni "a priori materiale" e "a priori sintetico" (usate con parsimonia dallo stesso Husserl) rimandino in realtà a un significato di a priori - quello fenomenologico - sostanzialmente differente da quello kantiano. Si tratterà quindi di mostrare come tale significato, pur includendo l'idea di una legalità necessaria e apodittica, nel caso della fenomenologia in realtà non solo non contraddica bensì confermi una prospettiva radicalmente empirista. Tale prospettiva sembra essere in qualche misura già racchiusa nelle due domande che la fenomenologia considera fondamentali. La prima domanda è che cosa è dato? La seconda domanda è che cosa è effettivamente contenuto nel dato?

\section{PAROLE-CHIAVE}

Husserl; Empirismo; A priori materiale; A priori sintetico; A priori fenomenologico

\section{ABSTRACT}

In this essay I will support the thesis according to which Husserl's perspective goes in the direction of an extremely sophisticated empiricism because at the same time radical but not reductionist. A form of empiricism, that is, which while aiming at a consistent revision of

\footnotetext{
${ }^{1}$ E-mail: roberta.lanfredini@unifi.it
} 


\section{Aoristo)))))}

International Journal of Phenomenology, Hermeneutics and Metaphysics

classical empiricism, never deviates from its general intent. One of the main objections that can be addressed to this reading is that the use of the notion of a priori, more particularly the notion of material a priori, appears to be both historically and theoretically contrary to an empiricist perspective. This objection can be largely reduced once we understand how the expressions "a priori material" and "a priori synthetic" (used sparingly by Husserl himself) actually refer to a meaning of a priori - the phenomenological one - substantially different from that Kantian. It will therefore be a question of showing how this meaning, while including the idea of a necessary and apodictic legality, in the case of phenomenology in reality not only does not contradict but rather confirms a radically empiricist perspective. This perspective seems to some extent already contained in the two questions that phenomenology considers fundamental. The first question is what is given? The second question is what is actually contained in the data?

\section{KEYWORDS}

Husserl; Empiricism; Material a priori; Synthetic a priori; Phenomenological a priori

\section{GENERALITÀ E INDUBITABILITÀ DEL DATO}

Nella prospettiva fenomenologica la nozione di dato ha carattere centrale, primitivo e assolutamente prioritario. Una delle parole che ricorrono maggiormente nei testi husserliani è, infatti, datità (Gegebenheit). Tre sono le tesi fondamentali che ruotano intorno a tale nozione: la tesi della generalità, la tesi della indubitabilità e la tesi della analizzabilità del dato. In questo paragrafo farò accenno solo alle prime due. La terza tesi, comprensiva della nozione di a priori materiale, verrà introdotta nel paragrafo successivo.

La nozione fenomenologica di dato è da intendersi in senso generalissimo e non limitato alla sfera della datità percettivo-esperenziale. In tale prospettiva risulta quindi dato non solo ciò che si manifesta nella percezione, ma anche ciò che si manifesta nell'immaginazione, nella fantasia e nel ricordo; sono ancora dati, inoltre, gli oggetti culturali, estetici, sociali, e così via, ma anche gli oggetti non intuitivi del pensare formale e puramente concettuale.

In questo senso, la critica alla nozione di dato puro, neutrale e indipendente da ogni tipo di schema concettuale, che tanto peso avrà poi nella riflessione epistemologica successiva all'empirismo logico, sembra: non essere qui del tutto pertinente: l'intento della fenomenologia, infatti, sembra essere non tanto quello di mettere a fuoco dati prioritari nel senso di neutrali e immodificabili ${ }^{2}$, quanto quello di analizzare il dato - qualsiasi dato, anche il "dato"teorico - esattamente come esso si dà nella sua evidenza e nella sua purezza. Il termine "puro", che sempre si associa all'evidenza fenomenologica, sembra avere una nota più privativa che positiva e qualificante: la descrizione del dato in quanto dato, descrizione che costituisce l'essenza della fenomenologia, ha infatti il compito non solo di rilevare ciò che si dà

\footnotetext{
${ }^{2}$ Quando Husserl interpreterà la percezione come fondante farà riferimento a un tipo di fondazione genetica e non statica: l'idea è che ogni datità sia radicata in ultima analisi nella percezione, non che ogni datità sia riducibile a dati percettivi.
} 


\section{Aoristo)))))}

\section{International Journal of Phenomenology, Hermeneutics and Metaphysics}

nella sua piena evidenza, ma anche esattamente come si dà, o come si manifesta, a esclusione di tutto ciò che effettivamente non si dà. La purezza della descrizione sembra riferirsi a quest'ultimo requisito.

È effettivamente compresa nella nozione di datità fenomenologica non solo la sua generalità ma anche il possesso di una modalità determinata. Dato è ciò che si dà nei modi in cui si dà e, d'altro canto, non esiste dato che risulti svincolato da certi modi del darsi. Questi modi risultano, almeno per Husserl, sempre e totalmente determinabili e esplicitabili. I modi in cui il fenomeno si dà possono riguardare elementi qualitativi: un dato è percepito, immaginato, pensato, desiderato; è appreso come chiaro o come oscuro, come dentro o come fuori del raggio dell'attenzione. Oppure legati alla messa a fuoco di alcuni aspetti dell'oggetto piuttosto che altre: l'oggetto percepito sarà percepito da una certa angolatura piuttosto che da un'altra angolatura; l'oggetto immaginato avrà alcune determinazioni e non altre (Edipo potrà essere inteso come l'assassino di Laio o come l'amante di Giocasta), le entità astratte saranno determinate in certi modi piuttosto che altri (lo stesso triangolo potrà essere determinato come triangolo equilatero e come triangolo equiangolo), e così via.

Il dato in quanto tale risulta inoltre, secondo Husserl, del tutto indubitabile. Sembra non esistere, nella prospettiva fenomenologica, una qualche utilizzazione di argomenti di tipo scettico. Ciò che lo scettico mette in dubbio, nel contestare l'affidabilità dei sensi, è la validità stessa della nozione di fenomeno: i sensi sono inaffidabili perché incompleti e parziali (la percezione di una moneta non sarà mai perfettamente tonda, ma ellittica, tondeggiante, piatta a seconda dell'angolatura); fuorvianti (come nell'illusione: il bastone immerso nell'acqua sembra spezzato, l'asfalto nella calura sembra bagnato); erronei (come nella allucinazione: posso vedere oggetti che non esistono); ambigui (come in qualsiasi caso di figura gestaltica) incapaci di auto-giustificazione (come nel sogno: potrei sognare e nel sogno non sapere che sto sognando). La risposta fenomenologica allo scettico sta tuttavia nel mostrare come del dato, nei modi ed esattamente nei limiti in cui esso è dato (della moneta percepita da quel determinato punto di vista, del bastone percepito come spezzato nell'acqua, dell'oggetto allucinato, della figura vista come anatra oppure come coniglio, degli eventi sognati pur senza sapere che si sta sognando) non è affatto lecito dubitare. Nella fenomenologia, quindi, non si dubita mai, nel senso in cui ne dubita lo scettico, della legittimità dei sensi ${ }^{3}$.

Io mi posso ingannare sull'esistenza dell'oggetto della percezione, ma non sul fatto che io lo percepisco come determinato in questo o in quest' altro modo, e che esso, nell'intenzione di questo percepire, non è un oggetto totalmente diverso, ad esempio un abete invece di un maggiolino. Questa evidenza nella descrizione determinante, o nell'identificazione e nella differenziazione reciproca degli oggetti

\footnotetext{
${ }^{3} E^{\prime}$, questo, uno dei punti che distanziano maggiormente il metodo utilizzato dalla fenomenologia dal metodo cartesiano che, com'è noto, prende le mosse proprio da un'istanza scettica.
} 


\section{Aoristo)))))}

International Journal of Phenomenology, Hermeneutics and Metaphysics

intenzionali come tali, benché abbia, come è facile comprendere, i propri limiti, è tuttavia un'evidenza vera e autentica. Anzi, senza di essa sarebbe senz'altro inutile anche la tanto famosa evidenza della percezione interna con la quale essa viene solitamente confusa, ogni volta che si intende la percezione "interna" come percezione di vissuti intenzionali; non appena ha inizio un discorso espressivo e viene compiuta la distinzione descrittiva tra vissuti appresi nella percezione interna, quest'evidenza è già presupposta, in quanto non è addirittura possibile descrivere e distinguere $\mathrm{i}$ vissuti intenzionali senza riferirsi ai loro oggetti intenzionali (HUSSERL, 1968, p.470) ${ }^{4}$.

Il dubbio metodico di tipo cartesiano viene sostituito da meccanismi che Husserl denomina riduttivi. Le principali riduzioni di cui la fenomenologia fa uso sono sostanzialmente due: chiameremo la prima riduzione riflessiva ${ }^{5}$, a sua volta articolata in una riduzione noetica e in una riduzione noematica. La seconda riduzione è la riduzione eidetica. Stando al primo corno della riduzione riflessiva, quello noetico, non esiste dato (o fenomeno, o oggetto) che non sia inteso. $E^{\prime}$ effettivamente contenuto nella nozione di dato il rimando a uno stato di coscienza, a un vissuto intenzionale: ogni dato è, necessariamente, relativo a un' intenzione corrispondente ${ }^{6}$.

Il principio stando al quale non si danno oggetti che non possano essere intesi è un principio che nella fenomenologia non trova eccezioni. In ambito descrittivo, ciò rimanda alla impossibilità di praticare una analisi noematica senza connetterla in modo essenziale a una indagine noetica. Tale impossibilità è dovuta, tuttavia, non al fatto che l'oggetto fenomenologico sia effettivamente contenuto nello stato di coscienza, bensì al fatto che nell'oggetto fenomenologico sia effettivamente contenuto il rimando a uno stato di coscienza: il rosso che sento rinvia necessariamente a una determinata sensazione di rosso; la sedia che vedo rinvia necessariamente a una determinata percezione di quella sedia; la proprietà pensata rinvia necessariamente a una determinata modalità del pensiero, e così via7.

È esattamente la constatazione - per Husserl cruciale - del fatto che nel dato sia effettivamente contenuto non il vissuto bensì il rimando al vissuto, che lo legittima a

\footnotetext{
${ }^{4}$ In tale prospettiva la percezione per così dire "autentica" non si differenzia, dal punto di vista fenomenologico-descrittivo, dalla illusione o dalla allucinazione. Anche l'atto di percezione allucinatoria gode infatti di quel carattere d'atto "posizionante" proprio, secondo Husserl, di tutte le percezioni. D'altro canto, l'oggetto allucinato, ci apparirà "in carne ed ossa" al pari di qualsiasi oggetto percepito. Tutto ciò contrariamente a quanto sostenuto, ad esempio, da Searle (1985, p. 45).

${ }^{5}$ L'espressione non è husserliana. Più usata da Husserl è l'espressione riduzione trascendentale che però sembra essere, come la nozione di a priori sintetico, in larga parte fuorviante se collocata in un quadro di riferimento di tipo fenomenologico.

${ }^{6}$ Per Husserl non vale tuttavia, contrariamente a quanto sostenuto da Brentano, il viceversa: esistono infatti Erlebnisse che non sono intenzionalmente diretti verso fenomeni o dati. Esistono infatti Erlebnisse non diretti verso oggetti, quindi non intenzionali, primi fra tutti le sensazioni.

${ }^{7}$ Il porre l'accento più sulla nozione di rimando che sulla nozione di intenzionalità, comporta considerare come evidenza prioritaria non tanto l'evidenza dei dati soggettivi, che risulta in realtà analiticamente derivata, quanto l'evidenza dei dati oggettuali, degli oggetti cioè a cui i vissuti sono intenzionalmente rivolti. In questo senso sembra fortemente inadeguato parlare di idealismo fenomenologico, se non altro per la valenza metodologica piuttosto che ontologica che il concetto di riduzione assume nella fenomenologia.
} 


\section{Aoristo)))))}

\section{International Journal of Phenomenology, Hermeneutics and Metaphysics}

parlare di "enigma della trascendenza" 8 . Il fatto che per Husserl (contrariamente a Hume, per esempio) l'oggetto inteso non sia effettivamente contenuto nello stato di coscienza (il canto della cantante, dice Husserl, è distinto dall'atto del cantare), pur essendo ad esso essenzialmente vincolato (non esiste canto della cantante che prescinda dall'atto del cantare), comporta la distinzione fra due distinti ambiti oggettuali: l'ambito degli oggetti immanenti (gli stati di coscienza, siano essi intenzionali o non intenzionali, insieme a ciò che in essi è effettivamente contenuto) e l'ambito degli oggetti trascendenti (gli oggetti intesi insieme a ciò che in essi è effettivamente contenuto). I due tipi di oggetti appartengono a regioni disgiunte e hanno quindi caratteristiche essenzialmente diverse. La prima di tali caratteristiche risiede nel fatto che gli oggetti immanenti sono per Husserl dati adeguatamente, nel senso di a-prospettici: essi si danno cioè in modo completo e per così dire "senza residui". Al contrario, gli oggetti trascendenti sono per lo più inadeguati, nel senso di incompleti, essenzialmente prospettici e unilaterali. Adeguati saranno quindi oggetti come la sensazione di rosso, l'udire il suono di un violino o l'immaginare il dio Giove; inadeguati saranno oggetti come il rosso visto, il suono eseguito dal violino, il dio Giove immaginato. Che l'immanenza possa a sua volta essere trattata come ambito oggettuale distinto è ancora una volta garantito dal principio di intenzionalità: gli oggetti immanenti sono infatti, esattamente come gli oggetti trascendenti, oggetti di ulteriori stati intenzionali, più specificamente stati di coscienza riflessivi.

La trascendenza fenomenologica, o trascendenza noematica, viene concepita da Husserl come polo unitario in grado di racchiudere sinteticamente tutti i possibili e indefiniti modi di datità e tutte le possibili determinazioni dell'oggetto, in un processo che è per essenza senza fine.

Nessuna percezione di cosa è definitivamente conclusa, resta sempre spazio per nuove percezioni che determinerebbero più precisamente le indeterminatezze e riempirebbero ciò che è vuoto. Ogni sviluppo arricchisce lo statuto di determinazione del noema della cosa che ininterrottamente appartiene alla medesima cosa X. Ł̀ un'evidenza eidetica che ogni percezione e molteplicità percettiva può essere ampliata per cui il processo è senza fine; quindi nessun afferramento intuitivo dell'essenza della cosa può essere così completo da non ammettere una successiva percezione che vi aggiungerebbe qualcosa di noematicamente nuovo (HUSSERL, 1976/2002, p.327).

Questa apertura, che d'altro canto ruota intorno a una sintesi unitaria e incessante dei possibili modi di apparizione dell'oggetto, è indicativa della essenziale e costitutiva inadeguatezza dell'oggetto che trascende il contenuto del vissuto, oggetto che abbiamo visto essere inadeguato, prospettico, unilaterale. Vedremo in seguito come debba essere interpretata la possibilità a cui qui ci si riferisce.

\footnotetext{
${ }^{8}$ Si veda, a questo proposito (HUSSERL, 1950/1988).
} 


\section{Aoristo)))))}

International Journal of Phenomenology, Hermeneutics and Metaphysics

La seconda riduzione, quelle eidetica, permette di rispondere più esaustivamente a una delle domande che abbiamo visto caratterizzare l'intento fenomenologico, cioè che cosa è effettivamente contenuto nel dato? Il dato concreto, per Husserl, è infatti non solo indubitabile ma anche complesso, affatto amorfo e indifferenziato. Esso risulta, insomma, dotato di struttura, e di una struttura autonoma e indipendente.

\section{LA STRUTTURA DEL DATO: ASTRAZIONE E IDEAZIONE}

Il fatto che la nozione fenomenologica di dato abbia carattere primitivo e prioritario non esclude né la sua complessità né il suo sottostare a una legalità. Ciò che si dà risulta infatti dotato di una struttura complessa per almeno due motivi fondamentali: il primo è che qualsiasi dato è un'essenza, il secondo è che il dato, inteso come ciò che si manifesta, è composto da parti fra loro connesse da leggi necessarie.

La prima affermazione rimanda alla nozione di riduzione eidetica o ideazione, la seconda alla teoria dell'intero e delle parti. Il concetto di a priori materiale pertiene specificamente a questa seconda teoria, la quale risulta tuttavia comprensiva della prima. Sarà quindi, innanzi tutto, il concetto di ideazione a dover essere introdotto $\mathrm{e}$ chiarito. A tal fine, ci sarà utile prendere le mosse da una questione che riveste importanza sia dal punto di vista storico filosofico sia dal punto di vista specificamente teorico. La questione riguarda la critica alla teoria dell'astrazione formulata (in modo del tutto autonomo) sia da Cassirer in Sostanza e funzione sia da Husserl nella Seconda ricerca logica e poi in Idee. Tale critica riguarda la teoria dell'astrazione nella sua duplice veste di teoria essenzialista e di teoria empirista dell'astrazione ${ }^{9}$.

Con il termine astrazione sia Cassirer sia Husserl intendono un processo conoscitivo fondato su un meccanismo che procede per negazione, o per esclusione, di certe caratteristiche, a partire da una molteplicità data. Nella astrazione empirista, il concetto sorge sulla base della rinuncia a certe note individuali (quelle eterogenee) a favore di altre note individuali (quelle comuni). Così facendo, il meccanismo astrattivo "[...] isola i tratti affini da ogni mescolanza con elementi eterogenei" (CASSIRER, 1973, p, 12) ${ }^{10}$. Nella versione metafisico-sostanzialista della astrazione (quella, ad esempio, di stampo aristotelico), il meccanismo procede ancora una volta per via privativa, solo che la rinuncia a certe note caratteristiche è questa volta determinata non tanto dall'individuazione di note comuni nella mescolanza con elementi eterogenei, quanto dalla individuazione delle note essenziali in una mescolanza con elementi inessenziali. Si costituisce così, sulla stessa base della

\footnotetext{
${ }^{9}$ La critica che Husserl rivolge alla teoria empirista dell'astrazione nella Seconda Ricerca logica è in realtà più articolata e dettagliata, in quanto prende in considerazione le varie teorie dell'astrazione: la teoria dell'attenzione di Mill, la teoria della rappresentanza di Locke e di Berkeley,la teoria della distinctio rationis di Hume (HUSSERL, 1984).

${ }^{10}$ Sulla relazione fra astrazione empirista, ideazione fenomenologica e funzione cassireriana e, più in generale, sulla interpretazione fra fenomenologia e neokantismo questa interpretazione diverge drasticamente da quella espressa da Friedman (2004).
} 


\section{Aoristo)))))}

International Journal of Phenomenology, Hermeneutics and Metaphysics

astrazione, una gerarchia concettuale che dalla molteplicità sensibile risale alla specie e dalla specie risale al genere, gerarchia in cui si realizza una progressiva identificazione dell'essenza della cosa. In questo caso, la formazione del concetto spoglia la molteplicità sensibile delle caratteristiche non tanto eterogenee quanto contingenti e non essenziali.

Nominalismo concettuale e realismo concettuale condividono, secondo Cassirer, la stessa logica della formazione del concetto. L'idea è infatti che in entrambi i casi le nozioni astratte possano essere ricavate dallo specifico contenuto di ciò che si dà in quanto sua parte costitutiva costante (nel caso dell'astrazione empirista comune ma individuale, a scapito delle note eterogenee o dissimili; nel caso della astrazione metafisico-sostanzialista comune perché essenziale, a scapito delle note inessenziali). "La contesa fra nominalismo e realismo - afferma Cassirer concerne solo la questione della realtà metafisica dei concetti, mentre non viene preso in considerazione il problema della loro valida definizione logica"(HUSSERL, 1984, p.17). E il problema della valida definizione logica sta, secondo Cassirer, nell' intendere il concetto come parte costitutiva comune di una serie di singole cose omogenee e simili. Nel caso della logica astrattiva, infatti, “[...] il concetto non si oppone come alcunché di estraneo alla realtà sensibile, ma forma una parte di questa stessa realtà, un estratto di ciò che in essa è direttamente contenuto" (HUSSERL, 1984, p.12).

Il contenuto che in tal modo si ottiene è rigorosamente della stessa qualità e natura degli oggetti medesimi dai quali esso è stato separato: esso indica una proprietà che certamente non sussiste isolata in generale, ma tuttavia può essere sempre indicata in questi oggetti come elemento costitutivo di essi e possiede quindi un' esistenza concreta. Secondo questo modo di pensare, il concetto è la "rappresentazione" di ciò che è comune: è la sintesi di quei singoli caratteri che appartengono in modo uniforme a determinate classi di oggetti"(HUSSERL, 1984, p.260).

La critica che Cassirer rivolge alla logica della formazione del concetto astrattiva è sostanzialmente basata sulla constatazione della arbitrarietà della scelta dei criteri mediante i quali una cosa viene fatta cadere sotto un certo concetto. Individuare la parte costitutiva comune all'interno di una molteplicità sensibile comporta dichiarare simili gli oggetti di tale molteplicità. Il problema è tuttavia che la domanda "simili rispetto a cosa?" risulta essere una domanda non univocamente determinata ma, al contrario, intrinsecamente arbitraria.

Questa sintesi, che collega e unifica due situazioni separate nel tempo, non ha negli stessi contenuti confrontati alcun diretto connotato sensibile. A seconda della maniera e della direzione in cui questa sintesi avviene, il medesimo materiale sensibile può invece essere compreso in forme concettuali molto diverse. 


\section{Aoristo)))))}

International Journal of Phenomenology, Hermeneutics and Metaphysics

Si riconosce in questi rapporti che il vero difetto della teoria della astrazione consiste nell'unilateralità con cui dal gran numero di possibili principi di coordinazione logica si sceglie soltanto il principio di somiglianza. In verità risulterà che una serie di contenuti, per dirsi compresa e ordinata, può essere disposta secondo i punti di vista più diversi, a condizione soltanto che nella costruzione della serie il punto di vista assunto come guida venga mantenuto invariato nella sua specifica natura qualitativa (HUSSERL, 1984, p. 24-25).

L'arbitrarietà della nozione di similarità come criterio di formazione del concetto può essere arginata solo se supportata da una visione radicalmente sostanzialistica. Solo tale visione è infatti in grado di limitare la soggettività intrinseca in ogni criterio contenutistico mediante l'utilizzazione di concetti sostanziali, che rimandano cioè alla struttura specifica dell'essere. Tale visione risulta tuttavia, per Cassirer, totalmente inaccettabile ${ }^{11}$.

La critica alla oggettività della nozione di similarità, critica che attraverserà tutta l'epistemologia da Popper (1970) ${ }^{12}$ in poi si fonda, per Cassirer, sulla idea che la teoria astrattiva della formazione del concetto, proprio in quanto teoria contenutistica, è destinata inevitabilmente alla arbitrarietà e quindi a una logica della formazione del concetto inadeguata. Il carattere unilaterale e arbitrario della domanda "simili rispetto a cosa?" deriva quindi in modo diretto dalla impostazione contenutistica del problema: la domanda è non univocamente determinata proprio in quanto sottintende una caratterizzazione qualitativa della nozione di concetto. Teoria contenutistica e teoria astrattiva della formazione del concetto vengono quindi, per Cassirer, a convergere nella incapacità di soddisfare ciò che ci aspettiamo da un concetto scientifico e cioè che "[...] in luogo della indeterminatezza e ambiguità del contenuto rappresentativo, esso instauri una netta e univoca determinatezza" (CASSIRER, 1973, p.16).

Sulla base di questa cruciale constatazione, Cassirer perviene a una teoria relazionale e non contenutistica della formazione del concetto e a una codificazione del concetto stesso come funzione, come ordinamento, teoria cha ha avuto grande

\footnotetext{
${ }^{11}$ La critica cassireriana alla teoria della astrazione si rivolge prevalentemente alla teoria empirista della astrazione, cosa che si verifica anche nel caso di Husserl. Per quanto riguarda la teoria essenzialista dell'astrazione, essa viene, data la tradizione filosofica a cui egli si riferisce, facilmente liquidata Cassirer. Molto più complessa è la relazione fra fenomenologia e essenzialismo, data la piena accettazione, da parte di Husserl, di una certa forma di essenzialismo, come fra poco vedremo. II punto è, tuttavia, che tale accettazione ha ben poco a che fare con l'adesione alla tradizionale teoria essenzialista cui Cassirer fa riferimento.

${ }^{12}$ Com'è noto, Popper critica la tesi del primato (sia logico sia psicologico) delle ripetizioni, tesi che costituisce la base del modello induttivo di inferenza scientifica. Tutte le ripetizioni che esperiamo risultano infatti essere approssimative, nel senso che la ripetizione $B$ di un evento $A$ sarà simile ad A sotto certi aspetti e non sotto altri aspetti. Al limite, afferma Popper, "dato un qualsiasi gruppo o insieme finito di cose, possiamo sempre, con un po' di ingegnosità, trovare punti di vista tali che tutte le cose che appartengono a quell'insieme siano simili (o parzialmente simili): ciò significa che di una ripetizione qualsiasi si può dire qualsiasi cosa, e ciò mostra inoltre quanto sia ingenuo considerare le ripetizioni come qualcosa di definitivo, di dato" (POPPER, 1970, p. 477). La critica alla similarità come base delle asserzioni scientifiche costituirà poi una delle conferme al relativismo epistemologico sostenuto da Feyerabend.
} 


\section{Aoristo)))))}

\section{International Journal of Phenomenology, Hermeneutics and Metaphysics}

ripercussione non solo in tutto l'empirismo logico, ma anche in gran parte della cosiddetta nuova filosofia della scienza.

La tradizione che dal neokantismo di Cassirer si riversa su alcune fondamentali istanze dell'empirismo logico per approdare infine ad alcuni esiti estremi della nuova filosofia della scienza, come quelli espressi da Feyerabend, sembra infatti condividere una idea di fondo: tale idea è che il contenuto, quindi anche l'osservazione comprensiva degli elementi qualitativi che inevitabilmente la accompagnano, giochi un ruolo nel processo conoscitivo solo se inserito e strutturato in schemi concettuali in grado di oggettivarlo. La teoria dell'astrazione mette in ombra esattamente questo aspetto, “[...] confondendo le forme categoriali sulle quali si fonda ogni determinatezza del contenuto della percezione con parti di questo contenuto medesimo"13.

Le qualità sensibili delle cose diventano oggetto della fisica in quanto si convertono in una determinatezza seriale. Da somma di proprietà la "cosa" diventa ora un complesso di valori matematici, stabiliti in base a una scala di confronto. I diversi concetti della fisica determinano ciascuno per sé una scala di tal genere e rendono così possibile una intima connessione e coordinazione degli elementi del dato. Il caos delle impressioni si trasforma in un sistema di numeri; ma questi numeri ricevono la loro denominazione e quindi il loro significato specifico solo dal contenuto dei concetti fondamentali, teoreticamente stabiliti come unità di misura universalmente valide. Solo in questo nesso logico si comprende il valore "oggettivo" che va riconosciuto alla trasformazione dell'impressione in "simbolo" matematico. Certamente, nella designazione simbolica è cancellata la particolare natura dell'impressione sensoriale; viene però mantenuto e messo in particolare evidenza tutto ciò che la caratterizza come termine del sistema. Il simbolo possiede il correlato perfettamente valido non in alcun elemento costitutivo della percezione stessa, bensì nella connessione regolare che sussiste fra gli elementi di essa; questa connessione regolare che sussiste fra gli elementi di essa; questa connessione però è ciò che si rivelerà in modo sempre più chiaro come il vero nucleo del concetto della stessa "realtà" empirica. [...] La materia della percezione non viene soltanto in un secondo tempo colata in una qualche forma concettuale; bensì il pensiero di questa forma costituisce il presupposto necessario per poter riferire ad essa qualsiasi predicato e determinazione concreta (CASSIRER, 1973, p. 203-204).

Proprio negli anni in cui Cassirer metteva a punto Sostanza e funzione, Husserl prendendo le mosse (e in modo altrettanto polemico) dalla teoria dell'astrazione,

\footnotetext{
${ }^{13}$ Cassirer (1973), p. 26. Naturalmente diversi sono i modi in cui intendere da un lato l'influenza della concettualizzazione nella osservazione (problema della dicotomia teorico-osservativo); dall'altro la natura degli schemi concettuali (critica al sintetico a priori e problema dello statuto dei principi teorici). Per entrambi gli aspetti si veda Parrini (2002). II filo rosso che attraversa questa lunga e per molti aspetti eterogenea tradizione sembra trovare un decisivo momento di discontinuità nella epistemologia di Kuhn, fondata sulla nozione di esemplare, e nel modello reticolare proposto da Mary Hesse. In questi due autori, infatti, ciò che muta è esattamente il modo di intendere lo schema concettuale in relazione all'aspetto contenutistico e osservativo. $\mathrm{Si}$ veda, a tale proposito, Lanfredini (1988), Parrini (1995).
} 


\section{Aoristo)))))}

\section{International Journal of Phenomenology, Hermeneutics and Metaphysics}

approdava a un risultato teorico profondamente diverso, incentrato sulla teoria fenomenologica dell'ideazione. L'intento di Husserl era non tanto, tuttavia, quello fornire una logica adeguata (non arbitraria) del concetto, quanto quello di fornire una descrizione fenomenologicamente adeguata, una descrizione "dall'interno" per così dire, proprio di quella molteplicità sensibile da cui sia la teoria dell'astrazione sia la teoria cassireriana del concetto come funzione prendevano le mosse.

Ciò che la teoria dell'ideazione sembra proporre è, innanzi tutto, una esplicazione della nozione di dato e di ciò che in tale nozione risulta effettivamente, analiticamente contenuto.

L'ideazione risulta essere un meccanismo che gode infatti delle seguenti caratteristiche.

La prima è che non si tratta di un processo astrattivo. L'ideazione non procede infatti per esclusione o negazione di note caratteristiche a favore di altre; quindi astrae ma, appunto, idea e nel fare questo non si fonda su alcuna constatazione di comparazione o similarità. Qualsiasi dato intuito risulta infatti ideabile in modo immediato, senza che si verifichi la necessità di rispondere alla domanda "rispetto a quali criteri esso viene ideato?".

Riconosco questa carta come carta e come bianca, e porto con me a piena chiarezza il senso generale delle espressioni carta e bianco come tali, senza effettuare intuizioni di uguaglianza e comparazioni [...].

La concezione empirista, che vuole evitare l'assunzione di oggetti specifici rinviando alla loro estensione, è pertanto impraticabile. Essa non è in grado di dirci che cosa dia unità all'estensione. L'obiezione che segue chiarirà ancora di più questo punto. La concezione che contestiamo opera con "ambiti di somiglianza", ma prende troppo alla leggera la difficoltà costituita dal fatto che ogni oggetto appartiene ad una molteplicità di "ambiti di somiglianza". Ma che cosa distingue l'uno dall'altro questi stessi "ambiti di somiglianza"? Questa è la domanda a cui ora bisogna rispondere. Si comprende che senza l'unità già data delle specie sarebbe impossibile evitare un regressus ad infinitum. Un oggetto A è simile ad altri oggetti, agli uni dal punto di vista a, agli altri dal punto di vista $b$, ecc. Il punto di vista stesso non può tuttavia voler dire che vi è una specie che crea l'unità. Che cos'è dunque ciò che rende unitario l'ambito di somiglianza determinata dall'essere rosso di fronte a quello determinato della triangolarità? La concezione empiristica può dire soltanto: si tratta di somiglianze differenti (HUSSERL, 1984, p.385).

L'ideazione, così facendo, interviene anche in presenza di una sola nota caratteristica dell'oggetto e, al contempo, può intervenire su tutte le note caratteristiche dell'oggetto isolatamente considerate. La principale obiezione che Husserl rivolge alla teoria dell'astrazione empirista, la quale pretende di parlare di generalità o di specificità solo partendo da una messa a fuoco di elementi individuali, sembra, paradossalmente, proprio quella di non essere sufficientemente empirista. E il motivo di ciò risiede nel fatto che la teoria dell'astrazione non fornisce una 


\section{Aoristo)))))}

\section{International Journal of Phenomenology, Hermeneutics and Metaphysics}

adeguata descrizione e valorizzazione conoscitiva esattamente di quelli che sono i fondamenti della impostazione empirista, cioè da un lato la nozione di dato e dall'altro la nozione di uguaglianza e di similarità fra dati. Entrambe le nozioni sottintendono infatti, secondo Husserl, un processo ideativo, come vedremo immediatamente.

Per quanto riguarda il dato individuale esso non potrebbe essere inteso se non fosse ideato: l'individuo risulta infatti essere tale - individuato, appunto - solo in quanto elemento di un campo di possibili variazioni limitate da confini eidetici. Se così non fosse, esso sarebbe un "substrato informe", privo di qualsiasi determinazione intuitiva 14. Qualsiasi campo di possibili variazioni deve necessariamente fare perno su un individuo: questo è il senso che legittima la tesi stando alla quale l'essenza non può non fondarsi su una entità individuale. È d'altra parte altrettanto vero che nella determinazione stessa dell'individuale, il qui e ora ci è praticamente indifferente. Se nell'intenzione dell'individuale fossero infatti presenti tutte le determinazioni realmente individualizzanti, di fatto non potremmo intendere alcunché. Per poter percepire lo stesso attributo individuale in oggetti differenti è necessaria l'applicazione della riduzione eidetica e avere quindi una specifica essenza.

Qui c'è l'amico Giovanni e io lo chiamo Giovanni. Indubbiamente egli è individualmente determinato, a lui spetta di volta in volta un luogo determinato, un posto determinato nel tempo. Ma se queste determinazioni fossero co-intenzionate, il nome muterebbe il proprio significato ad ogni passo compiuto dall'amico Giovanni e ogni volta che lo chiamo per nome. Ben difficilmente si potrà asserire qualcosa di simile $o$ anche tentare una scappatoia affermando che il nome proprio è in realtà un nome generale: come se la generalità che esso possiede in rapporto alla varietà dei tempi, delle condizioni e degli stati dello stesso individuum cosale non differisse, nella propria forma, dalla generalità specifica dell'attributo cosale o dell'idea generica "cosa in generale".

Comunque, nell'osservazione attenzionale di una frazione o di un elemento caratteristico dell'oggetto il qui e ora ci è abbastanza spesso indifferente [...]. Quando si effettua intuitivamente la coscienza della generalità come astrazione vera ed autentica, è sicuramente dato alla coscienza l'oggetto individuale dell'intuizione fondante, benché esso non sia affatto intenzionato.

\footnotetext{
${ }^{14}$ Si veda Husserl (1950) §14. Quando Husserl parla della fenomenologia come scienza di essenze contrapponendola alle scienze di dati di fatto, la sua utilizzazione di termini del tutto tradizionali come "esemplificazione", "corrispondenza", "fondazione dell'essenza sull'individuo" nascondono in realtà uno scenario del tutto anomalo rispetto alla tradizione stessa. La relazione fenomenologica fra individuo e essenza può essere letta in modo molto più appropriato nei termini di una relazione di appartenenza dell'individuo a un campo di variazione eidetica. In questa stessa direzione, che poco ha a che fare con la tradizionale distinzione fra sensibile e intelligibile, può essere reinterpretata la nozione platonica di "unità nella molteplicità". Rimando, per un'analisi più dettagliata di questo punto, a Lanfredini (2004, p. 135-144) e a Lanfredini (2003, p. 100-108).
} 


\section{Aoristo)))))}

International Journal of Phenomenology, Hermeneutics and Metaphysics

Se confrontiamo due distinti fenomeni concreti separati della medesima "qualità", ad esempio del "medesimo" verde, sappiamo con evidenza che ogni fenomeno ha il suo verde. I due fenomeni non sono avviluppati tra loro come se avessero un elemento comune individualmente identico, lo "stesso" verde; il verde dell'uno è piuttosto realmente distinto da quello dell'altro come sono distinti gli interi concreti ai quali appartengono. Come potrebbero altrimenti esister configurazioni qualitative unitarie nelle quali la medesima qualità può presentarsi più volte e che senso avrebbe ancora parlare di estensione di un colore sulla totalità di una superficie? Ad ogni ripartizione geometrica della superficie corrisponde evidentemente anche una ripartizione del colore unitario mentre noi, ammettendo che il colore sia del tutto identico, possiamo dire e diciamo che "il" colore è sempre "lo stesso" (HUSSERL, 1984, p. 425).

Se l'individuo risulta quindi in un certo senso dato ("noi non siamo, dice Husserl, divenuti improvvisamente ciechi nei confronti dell'individualità"15), esso non è ancora di per sé inteso. Esiste, di conseguenza, una essenzialità insita nella singolarità in rapporto alle variazioni dello stesso individuum ed è questa essenzialità, situata in un territorio intermedio fra individuale e universale, a essere oggetto immediato di un atto di intuizione.

In modo analogo, l'uguaglianza o la similarità fra dati, ad esempio fra due sfumature di rosso, non può istituirsi senza che sia presupposta l'identità.

In effetti, ogni qualvolta vi è uguaglianza, vi è anche identità in senso vero e rigoroso. Non possiamo caratterizzare due cose come uguali senza indicare secondo quale rapporto esse lo siano. Secondo quale rapporto, abbiamo detto, e proprio in questo risiede l'identità. Ogni uguaglianza si riferisce ad una specie alla quale sottostanno gli elementi che entrano nella comparazione; e questa specie, a sua volta, non è qualcosa di meramente uguale, e non lo può essere, altrimenti sarebbe inevitabile un assurdo regressus in infinitum.

Se due cose sono uguali in rapporto alla forma, l'identico sarà la specie relativa alla forma; se sono uguali in rapporto al colore, esso sarà specie del colore, ecc. Certamente, non ogni specie si esprime univocamente nella parola, e può accadere così che manchi l'espressione opportuna del rapporto, sarà forse difficile indicarlo chiaramente; tuttavia, noi lo abbiamo di mira, ed è esso che determina il nostro discorso sull'uguaglianza. Naturalmente, voler definire l'identità, sia pure soltanto nel campo sensibile, essenzialmente come caso limite dell'uguaglianza ci sembrerebbe una distorsione della verità delle cose. L'identità è assolutamente indefinibile, ma non l'uguaglianza. L'uguaglianza è il rapporto di oggetti che sottostanno ad una e ad una stessa specie

Per questo non si può rispondere al problema di come si realizzi l'apparente rilevazione o distinzione del colore nell'oggetto colorato (o dell'oggetto colorato) ricorrendo alla rilevazione della somiglianza fra l'oggetto colorato e altri oggetti colorati. Infatti, sviluppando coerentemente questa spiegazione, si sarebbe ricondotti 


\section{Aoristo)))))}

\section{International Journal of Phenomenology, Hermeneutics and Metaphysics}

alla rilevazione di una somiglianza di questa somiglianza con altre (nell'esempio del colore: gruppi di somiglianza di somiglianze, come quelli sussistenti tra oggetti colorati); a questa somiglianza si dovrebbe poi applicare lo stesso principio esplicativo, ecc. (HUSSERL, 1984, p. 383-384; p. 469) ${ }^{16}$.

La proposta di Husserl, proposta che segna il superamento della concezione empirista dell'astrazione, si fonda quindi sulla constatazione che per parlare di dato, o di similarità fra i dati, è necessario presupporre un processo ideativo sottostante (HUSSERL, 1984, p. 393)17.

La seconda caratteristica è che ciò che l'ideazione esprime è non tanto una logica della formazione del concetto, quanto una esplicitazione della natura del dato (quindi della molteplicità sensibile, o più semplicemente dei fenomeni) per così dire dal suo interno. Per Husserl il dato, qualsiasi dato, è essenziale, laddove l'essenza può essere tecnicamente definita, all'interno della fenomenologia, come il campo di variazione eidetica di un individuo. Il meccanismo della riduzione eidetica (così come il cosiddetto a priori materiale, che come fra poco vedremo ne costituisce uno degli elementi fondamentali), è in realtà un meccanismo estremamente complesso. Il fondamento di tale complessità è comunque rintracciabile nella constatazione che, se privato della sua essenzialità (fenomenologicamente intesa come unificazione della possibilità delle sue variazioni), il dato non potrebbe essere discriminato; non potrebbe essere inteso. In questo senso, la teoria fenomenologica del dato ripropone una forma di essenzialismo. Il punto è, tuttavia, che si tratta di un essenzialismo che non si fonda su un meccanismo di tipo astrattivo e che quindi non può essere ricondotto a quel tipo di essenzialismo al quale la critica di Cassirer si rivolge. Il motivo di ciò sembra risiedere, in ultima analisi, nel fatto che l'ideazione è un processo che può essere intuitivo, immediato, non concettuale.

Ciò che Husserl sembra non condividere della teoria di Cassirer è, in conclusione, il suo fondamento kantiano, cioè l'idea che sia necessaria una (buona, adeguata, non arbitraria) teoria della formazione del concetto per avere una (buona, adeguata, non arbitraria) teoria del dato. Così facendo, la teoria dell'ideazione prende le distanze sia dal concetto inteso come esplicitazione delle caratteristiche comuni di una molteplicità sensibile data (teoria dell'astrazione), sia dal concetto inteso come individuazione delle relazioni ordinatrici del molteplice (teoria del concetto come funzione). L'ideazione predispone quindi, in ultima analisi, un modo di fare teoria della conoscenza che sembra superare la premessa condivisa sia dalla teoria dell'astrazione sia dalla prospettiva di Cassirer: la premessa cioè che sia

\footnotetext{
${ }^{16}$ Si tratta di un argomento per molti aspetti simile a quello proposto da Kuhn in riferimento alla sua nozione di esemplare e da Mary Hesse in riferimento alla sua proposta di un modello conoscitivo di tipo reticolare. Questo vale soprattutto se intendiamo la nozione di essenza fenomenologica non nei termini classici di una separazione fra sensibile e intelligibile, bensì nei termini di una esplicazione (non concettuale) del dato.

${ }^{17}$ Il che non significa tuttavia aderire, è importante sottolinearlo, né a una ipostatizzazione metafisica dell'essenza, secondo la quale la specie gode di una esistenza reale fuori dal pensiero, né a una ipostatizzazione psicologica dell'essenza, secondo la quale la specie gode di una esistenza reale nel pensiero. Si veda, per una presa di distanza da entrambe le posizioni, oltre che dal nominalismo. Seconda ricerca logica, §7,
} 


\title{
Aoristo)))))
}

\section{International Journal of Phenomenology, Hermeneutics and Metaphysics}

necessaria una buona logica del concetto per poter avere una buona teoria dell'oggetto. Ciò che essa intende fare, si potrebbe dire, è di permanere, al pari della teoria dell'astrazione e a differenza della teoria cassireriana, nel dato originario, ma con la pretesa di permanere interamente nel dato originario, senza astrarre certe note escludendone altre, come avviene nella teoria dell'astrazione. Tutti gli attributi dell'oggetto sono, in quanto intesi in modo determinato, il risultato di una riduzione eidetica.

Da questo punto di vista, la differenza sostanziale è non tanto quella fra teoria contenutistica e teoria strutturale della formazione del concetto, quanto quella fra teoria della formazione del concetto (sia essa contenutistica, come nella teoria dell'astrazione, oppure strutturale e formale, come nella prospettiva cassireriana) e teoria fenomenologica della ideazione. Non solo, quindi, come afferma esplicitamente Husserl, "[...] intuire non equivale a pensare", ma "lo scopo, la conoscenza vera è il pensiero che attinge evidenza dall' intuizione" (HUSSER, 1984, p. 440-441). L'unificazione eidetica non è, primariamente, una unificazione concettuale, bensì una unificazione in prima istanza intuitiva.

Si tratta di un'idea la quale più che contraddire la prospettiva di Cassirer (tutta incentrata sulla unificazione e identificazione concettuale), rende senz'altro più complessa e articolata la sua premessa iniziale (l'unificazione e identificazione del molteplice sensibile a prescindere dalla unificazione e identificazione concettuale).

\begin{abstract}
Risulta quindi [...] che ogni formazione di concetti è legata a una determinata forma di costruzione di serie. Diciamo concettualmente compresa e ordinata una molteplicità offerta dall'intuizione allorché i suoi termini non stanno l'uno accanto all'altro senza rapporti, ma derivano in successione necessaria da un determinato termine iniziale secondo una fondamentale relazione generatrice. L'identità di questa relazione generatrice, che viene mantenuta pur nel mutare dei singoli contenuti, è ciò che costituisce la forma specifica del concetto (CASSIRER, 1973, p. 25).
\end{abstract}

In conclusione: l'ideazione è un processo intuitivo comprensivo di un duplice movimento: quello che dall'individuo va all'essenza e il movimento inverso. Il carattere intuitivo comporta innanzi tutto l'istantaneità del passaggio da individuale a essenziale. In secondo luogo la non individuazione di criteri di unificazione o di similarità fra individuali: la discriminazione di una singolarità (come una determinata sfumatura di rosso cremisi) non comporta infatti la necessità di isolare criteri concettuali rispetto ai quali quella stessa singolarità possa essere identificata come tale. Infine, la relazione fra individuo e essenza non presuppone una visione dicotomica fra il piano della sensibilità e il piano della concettualizzazione. La relazione fra individuo e essenza si muove, come si verifica in gran parte delle distinzioni fenomenologiche, in un unico orizzonte ontologico: l'individuo è il perno intorno al quale ruotano tutte le sue possibili variazioni affinché rimanga lo stesso individuo; i confini eidetici sono, in modo speculare, i confini all'interno dei quali 


\section{Aoristo)))))}

International Journal of Phenomenology, Hermeneutics and Metaphysics

l'individuo può variare pur rimanendo tale. In questo senso sembra lecito sostenere che è effettivamente contenuto nel dato la sua essenzialità.

La terza caratteristica, strettamente connessa alle due precedenti, è che l'ideazione, al pari della astrazione e a differenza del concetto come funzione proposto da Cassirer, mantiene intatto il tratto contenutistico che caratterizza il dato, o il fenomeno, o la molteplicità sensibile. Così facendo, l'ideazione fenomenologica sembra inserirsi a pieno titolo nel solco della tradizione dell'empirismo classico, aderendo a una istanza empirista estremamente sofisticata e al tempo stesso radicale. Il carattere sofisticato di tale impostazione empirista risiede nella constatazione del fatto che il dato sottostà, per Husserl, a una legalità interna e necessaria. È esattamente questo tipo di legalità che viene racchiuso nell'espressione "a priori materiale".

\section{LA STRUTTURA DEL DATO:LA GERARCHIA EIDETICA E IL RAPPORTO DI FONDAZIONE}

Che la fenomenologia sia scienza di essenze non significa che tali essenze siano necessariamente degli universali. Al contrario, le essenze fenomenologiche sono sostanzialmente singolarità. D'altro canto, è lecito sostenere che in una prospettiva fenomenologica la nozione di singolarità non esclude affatto quella di eideticità. Le singolarità eidetiche a cui Husserl si riferisce sono infatti rigorosamente distinte dalle individualità o dati di fatto. Due sono gli argomenti che sembrano condurre Husserl all'ammissione della esistenza di singolarità eidetiche: il primo argomento rimanda alla constatazione del fatto che la nozione di dato comprende quella di idea; il secondo argomento rimanda alla constatazione del fatto che la nozione di dato comprende quella di legalità necessaria.

Il primo argomento risulta essere compreso nella critica alla teoria dell'astrazione, più specificamente nel riconoscimento che tale teoria non tiene conto della "astrazione" presente nel singolo dato. Non è affatto vero, infatti, che nell'osservazione del singolo intendiamo i momenti individualizzanti (temporalità, localizzazione, ecc.). Al contrario, come abbiamo visto, nella determinazione del singolo, il qui e ora ci è quasi sempre indifferente. Esiste quindi una specificità insita nel concetto di singolarità in rapporto alla varietà dei tempi, delle condizioni e degli stati dello stesso individuum e ciò rende perfettamente lecito parlare non solo di una generalità specifica ma anche di una singolarità specifica. La singolarità specifica, o singolarità eidetica, è il risultato diretto e immediato del processo ideativo. Essa risulta inoltre inserita, per sua stessa natura, in una gerarchia eidetica materiale, cioè contenutisticamente determinata.

Ogni essenza, sia essa materiale oppure vuota (cioè puramente logica), si inserisce in una gerarchia di essenze, in una gerarchia di specie e di generi. Questa gerarchia ha necessariamente due limiti che non coincidono mai. Scendendo, giungiamo alle ultime differenze 


\section{Aoristo)))))}

\section{International Journal of Phenomenology, Hermeneutics and Metaphysics}

specifiche o, come possiamo anche dire, alle singolarità eidetiche, salendo, attraverso le essenze di specie e di genere, giungiamo a un genere supremo. Le singolarità eidetiche sono essenze che hanno necessariamente sopra di sé essenze "più generali" come loro generi, mentre non hanno sotto di sé altre particolarizzazioni, rispetto alle quali sarebbero esse stesse specie [...]. Allo stesso modo, il genere supremo è quello che non ne ha un altro sopra di sé (HUSSERL, 1976, p. 32-33).

La gerarchia eidetica procede quindi dal genere sommo (ad esempio il genere qualità sensibile, o il genere figura spaziale) e da questa al genere (ad esempio il genere colore, o il genere estensione), dal genere passa alla specie (ad esempio la specie rosso, o la specie figura triangolare) e dalla specie alle ultime differenze specifiche (ad esempio quella particolare sfumatura di rosso carminio o quella particolare configurazione triangolare). Le relazioni "verticali" che vigono fra specie e generi e fra ultime differenze specifiche e specie, sono relazioni di inclusione.

Questi rapporti tra essenze, designati attraverso i termini di genere e specie (e non già rapporti di classi, ossia di insiemi), implicano che nell'essenza particolare sia "contenuta mediatamente o immediatamente" quella più generale, in un senso determinato da afferrare, conformemente alla sua peculiarità, nell'intuizione eidetica. [...]. Ciò che è eideticamente singolare implica dunque tutte le generalità che si trovano al di sopra di esso, e queste sono a loro volta gerarchicamente "una nell'altra", quella superiore sempre in quella inferiore (HUSSERL, 1976, p. 32-33).

Il rosso carminio include quindi il rosso in specie e quest'ultimo include il genere colore. Il tutto dominato dal genere materiale supremo qualità sensibile. La garanzia del fatto che effettivamente sussistano rapporti di fondazione fra generi, specie e ultime differenze specifiche si ricava, ancora una volta, dall'intuizione: nella percezione di una sfumatura di rosso carminio risulta infatti essere effettivamente contenuta l'essenza specifica del rosso e, ancora, l'essenza generale del colore. Se così non fosse, la percezione di quella particolare sfumatura di rosso sarebbe concretamente impossibile.

L'analisi non può quindi procedere ulteriormente:

[...] questa modalità non può essere ulteriormente descritta. Infatti, se si chiede che cosa differenzi le determinazioni essere un momento sensoriale nel modo della "qualità", non è possibile dare alcuna risposta ulteriore, noi non possiamo indicare una determinazione aggiuntiva che non includa il concetto di qualità: così come, se si chiede che cosa si dovrebbe aggiungere al colore, perché ne risulti la 


\section{Aoristo)))))}

International Journal of Phenomenology, Hermeneutics and Metaphysics

specie rosso, noi potremmo rispondere ancora soltanto il rosso (HUSSERL, 1984, p.40). ${ }^{18}$

Ogni individuo ha quindi come essenza un concreto, inteso come la singolarità eidetica corrispettiva.

Un'essenza non indipendente si dice astratto, una assolutamente indipendente si dice concreto. Un questo-qui, la cui essenza riempita è un concreto, si dice individuo (HUSSERL, 1976, p.25).

La coscienza della generalità specifica deve valere come un modo di "rappresentare" essenzialmente nuovo, non solo in rapporto alla rappresentazione delle singolarità individuali, ma anche perché esso porta alla coscienza un nuovo genere di singolarità: le singolarità specifiche (HUSSERL, 1984, p.420).

Genere

Specie

Ultima differenza specifica (singolarità) (qualità colore)

(Rosso)

Le relazioni "verticali" di inclusione materiale non sono tuttavia ancora sufficienti per mettere a punto in modo concreto una gerarchia eidetica materiale. Tali relazioni risultano infatti affiancate da relazioni "orizzontali" di fondazione (Fundierung), le quali sussistono fra quelle che Husserl denomina parti non indipendenti dell'oggetto, intese come differenti determinazioni del fenomeno. Husserl definisce come parte ciò che costituisce effettivamente un oggetto, considerando l'oggetto in sé e per sé, cioè facendo astrazione da tutti i nessi dei quali esso è naturalmente intessuto (in questo senso sarà parte dell'oggetto il fatto che sia

\footnotetext{
${ }^{18}$ Tutto ciò indica i confini di quella che Husserl definisce ontologia materiale o regionale. Al di sopra dei sommi generi ha infatti inizio l'ontologia formale, la vuota logica del "puro qualcosa", la quale di per sé non costituisce alcuna regione in senso proprio. II passaggio dall'ultima differenza specifica alla specie e dalla specie al genere viene denominato da Husserl generalizzazione, mentre il passaggio inverso implica una specializzazione. Generalizzazione e specializzazione non devono essere tuttavia confuse con la formalizzazione e saturazione. Nel primo caso si fa infatti riferimento a rapporti interni alla gerarchia materiale, mentre nel secondo si opera un passaggio dall'ontologia materiale all'ontologia formale, una sorta di "svuotamento" di contenuto (formalizzazione) o di "riempimento" di contenuto (saturazione, de-formalizzazione). La relazione fra ontologia materiale e ontologia formale non è di inclusione: le essenze formali non sono cioè contenute nelle singolarità specifiche nello stesso senso in cui lo sono le specie e i generi materiali.
} 


\section{Aoristo)))))}

\section{International Journal of Phenomenology, Hermeneutics and Metaphysics}

rosso e rotondo, mentre non è parte dell'oggetto che sia esistente o che sia qualcosa. Viene inoltre definita parte non indipendente di un oggetto ogni momento astratto di un intero, cioè ogni parte di esso che non può essere oggetto autonomo di una rappresentazione. Saranno quindi parti non indipendenti delle cosa fisica, ad esempio, il colore e la forma le quali necessitano per essenza (quindi a priori) di una connessione con altre parti: il colore, in altri termini, è destinato necessariamente a esistere come momento di un oggetto colorato, a diffondersi in una certa estensione. Tali parti o determinazioni dell'oggetto si dicono anche disgiunti, "[...] poiché nel loro contenuto non hanno nulla in comune" (HUSSERL, 1984, p 19-20). Verrà infine definita parte indipendente dell'oggetto ogni "pezzo" di un intero, cioè ogni parte di esso che può divenire oggetto autonomo di una rappresentazione ad esso rivolta ${ }^{19}$.

Affermazioni come "un colore non può essere senza una certa estensione", oppure "non vi è timbro senza durata" implicano così modalità di connessione fra contenuti non-indipendenti, modalità che si fondano sulla specificità essenziale delle parti che costituiscono l'intero. I momenti disgiunti del fenomeno non si danno, quindi, se non in connessione con altri momenti (e proprio per questo non possono essere rappresentati separatamente), a differenza di contenuti che anche se non di fatto possono in linea di principio essere svincolati da ciò che li circonda.

In questo senso sembra sia lecito sostenere che l'integrazione fra i momenti o parti non indipendenti soddisfa una legalità materiale necessaria: l'impossibilità di rappresentare un colore senza estensione è una impossibilità essenzialmente diversa dall'impossibilità (empirica, di fatto) che ho di rappresentare il dato visuale testa senza lo sfondo visuale da cui esso assume risalto.

Ancora una volta è la nozione di essere effettivamente contenuto a venirci in soccorso: è infatti effettivamente contenuto nel colore il suo diffondersi in una estensione (legame logico-materiale) mentre non è effettivamente contenuto in una determinata superficie colorata l'essere vincolata a un determinato sfondo (legame empirico). Nel primo caso "si tratta di differenze intrinseche, che si fondano nell'essenza pura delle cose": si tratta quindi di modi di connessione a priori nel senso che si fondano sulla specificità essenziale, sulla natura delle parti che costituiscono un intero.

Con la soppressione della qualità si sopprime inevitabilmente l'intensità e inversamente con la soppressione dell'intensità la qualità. Questo non è evidentemente un fatto empirico, ma una necessità a priori che si fonda sull'esperienza.

Noi possiamo rappresentarci un uomo con due teste, la parte superiore di un uomo collegata alla parte inferiore di un cavallo, o anche elementi particolari, una testa, un naso, un orecchio, di per sé stessi. È invece impossibile produrre l'immagine di un'idea "astratta", ad esempio separare l'idea di un movimento da quella di un corpo mosso.

Non bisogna poi confondere l'illimitata generalità delle leggi naturali con la generalità dell'essenza. La proposizione "tutti i corpi sono pesanti" non pone certamente nessuna determinata cosa come esistente nella natura; tuttavia, non

\footnotetext{
${ }^{19}$ Si veda, a questo proposito, Husserl (1984), §1-12.
} 


\section{Aoristo)))))}

\section{International Journal of Phenomenology, Hermeneutics and Metaphysics}

possiede l'incondizionata generalità delle proposizioni eidetico-generali, nella misura in cui, per il suo senso di legge naturale, implica sempre una proposizione di esistenza, e precisamente quella della natura stessa, della realtà spazio-temporale: tutti i corpo, nella natura, cioè tutti i corpi "reali" sono pesanti. Al contrario, la proposizione "tutte le cose materiali sono estese" possiede validità eidetica e può essere intesa come puramente eidetica, in quanto la posizione di esistenza riferita al soggetto viene messa da parte (HUSSERL, 1984, p. 22-23).

I rapporti di fondazione fra momenti o parti non indipendenti costituiscono quella logica materiale che prende il nome di a priori materiale.

Genere

(colore)

Specie

(rosso) $\leftarrow \rightarrow$

Ultima differenza specifica

(determinata triangolarità)

(estensione)

Specie

(forma triangolare)
Genere

Ultima differenza specifica

(rosso cremisi)

\section{CONCRETO}

I rapporti di fondazione, o di connessione materiale necessaria, sembrano inoltre godere delle seguenti caratteristiche.

Essi vigono solo fra generi, mentre sembrano non valere fra specie e tanto meno fra ultime differenze specifiche. Se è vero che colore si fonda sull'estensione, è altrettanto vero che nessuna particolare estensione è necessariamente $\mathrm{e}$ materialmente vincolata a una particolare sfumatura di colore, così come nessuna specie di colore si fonda necessariamente su una configurazione spaziale. Esiste tuttavia una fondazione indiretta in grado di connettere in modo necessario l'ultima differenza del colore al genere estensione, e viceversa, offrendo in tal modo alla gerarchia eidetica una compattezza che altrimenti rischierebbe di far cadere la singolarità eidetica nel baratro del puramente individuale e dell'indistinto.

Quando si parla di unitarietà della fondazione si vuol dire che ogni contenuto si trova, direttamente o indirettamente, in un rapporto di fondazione con ogni altro contenuto. Ciò può accadere in modo tale che tutti questi contenuto siano fondati gli uni negli altri, immediatamente o mediatamente, senza ricorsi esterni; o 


\section{Aoristo)))))}

\section{International Journal of Phenomenology, Hermeneutics and Metaphysics}

inversamente, essi fondano tutti insieme un nuovo contenuto, sempre senza ricorsi esterni (HUSSERL, 1984, p. 66).

L'assenza, a cui Husserl esplicitamente si riferisce, di "ricorsi esterni" rimanda alla seconda caratteristica: l'oggetto risulta unicamente dalle parti non indipendenti e dai rapporti di fondazione che vigono fra essi, senza tuttavia che risulti in alcun modo necessario né un substrato sostanziale né un qualche momento autonomo di unità responsabile delle relazioni necessarie fra i momenti di un intero. Qualsiasi contenuto o principio autonomo e per così dire "estraibile"viene con ciò bandito dalla costituzione fenomenologica dell'oggetto, almeno nella misura in cui questo termine assume una connotazione di necessarietà.

L'unità di estensione e di colore, di qualità e di intensità di suono, $\mathrm{o}$ l'unità tra la componente sensoriale di una percezione di cosa e quei momenti fenomenologici peculiari che la coscienza percettiva immette in essa e così via, si sostengono unicamente sulle fondazioni unilaterali e bilaterali, senza che dal loro essere insieme venga fondato un contenuto-forma (Forminhalt) autonomo, un autonomo momento di unità. Salta agli occhi in ogni caso il dato di fatto che ogni qual volta si possono esibire effettivamente nell' intuizione forme come momenti autonomi, ciò che viene connesso sono parti relativamente indipendenti le une rispetto alle altre; ad esempio i suoni nell'unità della melodia, i colori separati come frazioni nell'unità della configurazione cromatica, figure parziali nell'unità della figura complessa, ecc. Invano ci sforziamo invece di trovare nell'unità del fenomeno visivo, accanto ai contenuti-forma che conferiscono unità alla frazioni, anche contenuti che colleghino insieme i momenti non indipendenti, come il colore e l'estensione, oppure all'interno del colore la tonalità cromatica e la luminosità, all'interno dell'estensione il momento della forma e quello della grandezza (HUSSERL, 1984, p. 66).

Un qualche contenuto o momento di unità indipendente sembra quindi rintracciabile solo nella connessione di parti indipendenti dell'oggetto, come nel caso dei suoni nella unità di una melodia, o nel caso dei colori nella unità di una configurazione cromatica, in tutti quei casi cioè in cui si verifica la possibilità di estrapolare e astrarre una forma sensibile (una melodia, per esempio) dotata di autonomia. Sembra quindi lecito sostenere che una funzione di unificazione o un momento autonomo di unità sia rintracciabile solo nella connessione di parti indipendenti di un intero: le relazioni di connessione fra "pezzi" di un intero sono relazioni fattuali (fermo restando che una parte indipendente è comunque il risultato di rapporti di fondazione fra momenti); le relazioni di fondazione fra momenti sono, al contrario, essenziali e necessarie ${ }^{20}$.

\footnotetext{
${ }^{20}$ Questa osservazione sembra segnare una differenza cruciale fra fenomenologia e la teoria della Gestalt, che si occupa sostanzialmente del primo tipo di relazioni.
} 


\section{Aoristo)))))}

International Journal of Phenomenology, Hermeneutics and Metaphysics

Nella costituzione del dato non è effettivamente contenuta l'esistenza di un contenuto autonomo o estraibile. Il rapporto di fondazione, cioè l'a priori materiale fenomenologico, sembra non comportare né l'individuazione di una forma sensibile indipendente né l'esistenza di una funzione unificatrice di tipo concettuale o intellettuale ("l'unità - afferma Husserl - è un predicato categoriale"(HUSSERL, 1984, p. 70): sono infatti gli elementi del dato che, in modo del tutto intrinseco, si fondano gli uni negli altri dando luogo a interi indipendenti.

E non è forse un controsenso pretendere di collegare dei contenuti senza un legame? Naturalmente, tutto ciò è senz'altro giusto in rapporto ai contenuti che l'immagine presuppone. Ma quei contenuti di cui parliamo hanno molto a che fare l'uno con l'altro: essi sono anzi fondati l'uno nell'altro, e proprio perciò non hanno bisogno di vincoli e di legami, per essere messi in rapporto. Anzi, tutte queste espressioni non hanno propriamente per loro alcun senso. Dove non ha senso parlare di distinzione, è privo di senso anche il problema del modo in cui la distinzione può essere superata. [...] Ciò che unifica veramente ogni cosa, dovremmo dire propriamente, sono i rapporti di fondazione. Di conseguenza anche l'unità degli oggetti indipendenti si realizza solo mediante la fondazione (HUSSERL, 1984, p. 69).

La terza caratteristica della fondazione, strettamente vincolata alla prima, è che l'ontologia a cui essa dà luogo è costituita sì da essenze ma da essenze che oltre a essere singolari sono anche inesatte, fluenti, morfologiche. Le singolarità eidetiche sono infatti entità non deducibili dalla gerarchia eidetica e alla eideticità specificamente fenomenologica non corrisponde, quindi, alcun ideale matematizzante, alcuna idealizzazione nel senso delle scienze esatte o della logica pura. La fenomenologia, come Husserl ribadisce a più riprese, non è né una geometria né tanto meno una logica dei fenomeni ${ }^{21}$. Infatti nella geometria o nella matematica (almeno nella geometria e nella matematica che Husserl aveva in mente) "non rimane per principio più nulla di indeciso" o di indeterminato. Se la geometria, che Husserl vede come scienza eidetica ideale e materiale, partendo da pochi principi “[...] è in grado di ricavare in modo puramente deduttivo tutte le figure 'esistenti' nello spazio" e, così facendo può "[...] essere pienamente sicura di dominare col suo metodo veramente ed esattamente tutte le sue possibilità"(HUSSERL, 1976, p.173), ciò non si verifica affatto nella fenomenologia, la quale non determina esattamente, mediante deduzioni, il complesso delle entità di cui si occupa.

\section{LA MATEMATIZZAZIONE DELLA NATURA}

Per poter parlare di entità "non casualmente ma essenzialmente inesatte, e quindi non matematiche", il lavoro ontologico del fenomenologo risulta molto più

\footnotetext{
${ }^{21}$ Matematica e geometria si occupano entrambe, per Husserl, di idealità: idealità formali nel caso della matematica e materiali, oggetto cioè di intuizione, nel caso della geometria.
} 


\section{Aoristo)))))}

\section{International Journal of Phenomenology, Hermeneutics and Metaphysics}

simile alle descrizioni del botanico che alle descrizioni dello scienziato cosiddetto "esatto". La legalità essenziale della fenomenologia si distingue così dalla idealità delle scienze cosiddette esatte.

Con un po' di sottigliezza si dovrebbe distinguere tra separazione (o delimitazione) più netta $\mathrm{o}$ più fluida, e proprio nel senso empiricamente vago in cui nella vita comune si parla, ad esempio, di punte o di spigoli aguzzi, contrapponendoli a quelli smussati o addirittura arrotondati. È chiaro che le formazioni essenziali di tutte le datità intuitive come tali non vanno, per principio, sussunte sotto concetti "esatti" o "ideali" come quelli matematici. La forma spaziale dell'albero percepito come tale, assunta appunto così come si può trovare nella relativa percezione, come momento del suo oggetto intenzionale, non è una figura geometrica, non è qualcosa di "ideale" o di "esatto" nel senso della esattezza geometrica. proprio nel senso empiricamente vago in cui nella vita comune si parla, ad esempio, di punte e di spigoli aguzzi, contrapponendoli a quelli smussati o addirittura arrotondati. È chiaro che le formazioni essenziali di tutte le datità intuitive non vanno, per principio, sussunte sotto concetti "esatti" o "ideali" come quelli matematici. [...]. Le essenze colte nelle datità intuitive mediante una ideazione diretta sono essenze "inesatte" (HUSSERL, 1984).

D'altro canto, è lecito sostenere che

[...] il geometra non si interessa delle forme fattuali, che possono essere intuite dal punto di vista sensibile, come fa invece lo scienziato quando indaga descrittivamente la natura. Egli non foggia, come quest'ultimo, concetti morfologici di vaghi tipi di forme, tipi afferrati sul fondamento dell'intuizione sensibile e poi fissati, nella loro vaghezza, sul piano concettuale e terminologico. La vaghezza dei concetti, il fatto che le loro sfere di applicazione siano fluenti, non è una macchia che bisogna imputar loro poiché, nella sfera conoscitiva all'interno della quale vengono utilizzati, sono indispensabili, ossia, in tale sfera sono gli unici legittimi. Se dobbiamo dare una espressione concettuale conveniente alle datità intuitive delle cose con il loro caratteri essenziali intuitivamente dati, le dobbiamo assumere come esse si danno. Esse si danno appunto come fluenti, e le essenze tipiche possono essere afferrate in esse solo attraverso l'intuizione eidetica che procede attraverso analisi immediate. La geometria più perfetta e il più perfetto dominio pratico non possono aiutare lo scienziato che vuole descrivere la natura a esprimere (in concetti gometrici esatti) ciò che egli esprime in maniera semplice, comprensibile e del tutto appropriata con le parole: frastagliato, dentellato, lenticolare, ombrelliforme, ecc. - importanti concetti, che sono non casualmente, ma essenzialmente inesatti, $e$ quindi non matematici . I concetti geometrici sono concetti "ideali". Essi esprimono qualcosa che non si può "vedere"; la loro "origine" e 


\section{Aoristo)))))}

\section{International Journal of Phenomenology, Hermeneutics and Metaphysics}

quindi il loro contenuto sono essenzialmente diversi da quelli dei concetti descrittivi che non esprimono alcuna essenza "ideale" (HUSSERL, 1976, p. 176-177).

L'idealità può essere fenomenologicamente intesa come "forma limite", forma verso la quale "[...] tende, come un polo invariabile e insieme irraggiungibile, qualsiasi serie di perfezionamenti. In quanto ci interessiamo di queste forme ideali e ci impegnamo conseguentemente a determinarle e a costruirne di nuove sulla base di quelle che sono già state costruite, noi siamo "geometri""(HUSSERL, 1961, p.55).

La distinzione fra essenzialità e idealità costituisce il fulcro intorno al quale ruota la relazione fra scienza fenomenologico-descrittiva e scienza naturale matematica di stampo galileiano, oltre che uno dei temi centrali de La crisi delle scienze europee ${ }^{22}$. Si tratta della questione della matematizzazione, sia diretta sia indiretta, dei plena. Nella concezione galileiana la grande novità è costituita dalla concezione di questa idea di una totalità infinita dell'essere e di una scienza razionale che lo domina razionalmente. Questo mondo infinito, questo mondo di idealità è concepito in modo tale che i suoi oggetti non possono essere attinti singolarmente, imperfettamente e come casualmente dalla nostra conoscenza: esso può essere raggiunto soltanto da un metodo razionale, sistematicamente unitario - nel procedere infinito, infine, di ogni oggetto verso il suo pieno essere in sé.

Tuttavia, per Husserl (1961): “[...] l'idea galileiana è un'ipotesi, e un'ipotesi di un genere sorprendente" (p.71), una sorta di "splendido artificio". Si tratta ora di capire in che senso.

Il fatto è che i plena, cioè la componente più specificamente contenutistica dell'ontologia materiale (i suoni, i colori, le qualità tattili e olfattive) ${ }^{23}$ non sono direttamente quantificabili o matematizzabili.

Il motivo della impossibilità di una matematizzazione diretta dei plena sembra risedere nella loro natura non ideale, anche se essenziale. L'idea della applicazione di una scienza di idealità pure - come quelle della geometria o della matematica - al mondo delle datità sensibili sembra quindi incontrare, da questo punto di vista, un ostacolo difficilmente aggirabile.

Nel mondo circostante intuitivo, nella considerazione astrattiva delle forme spazio-temporali, noi esperiamo innanzi tutto "corpi" - non i corpi geometrico-ideali, bensì quei corpi che noi realmente esperiamo, provvisti di quel contenuto che è il reale contenuto dell'esperienza. [...] Le cose del mondo intuitivo e tutte le loro proprietà sono infatti immerse in generale nelle oscillazioni della mera tipicità; la loro

\footnotetext{
23 "Concretamente le forme empiriche reali o possibili ci sono date dapprima nell'intuizione empirica sensibile soltanto come "forme" di una "materia", di un plenum [Fülle] sensibile; cioè con ciò che si rappresenta nelle cosiddette qualità specifiche di senso, colore, suono, odore e simili, e secondo peculiari gradualità" (HUSSERL, 1961, p. 59).
} 


\title{
Aoristo)))))
}

\section{International Journal of Phenomenology, Hermeneutics and Metaphysics}

identità, il loro essere uguali-a-se-stesse e la loro temporanea permanenza nell'uguaglianza è soltanto approssimativa, proprio come la loro uguaglianza con altre (HUSSERL, 1961, p. 52, 55) ${ }^{24}$.

La datità sensibile, lo abbiamo visto, è caratterizzata dalla sua tipicità, dal suo carattere morfologico, vago. La riduzione eidetica, lungi dal contraddire questa idea, la conferma pienamente. Le invarianze che tale riduzione mette in evidenza sono infatti a loro volta essenze inesatte, tipiche, morfologiche e non certo idealità esatte nel senso della matematica e della geometria. L'idealità, intesa come forma limite "verso cui tende, come un polo invariabile e insieme irraggiungibile, qualsiasi insieme di perfezionamenti", sembra essere propria solo di una componente della gerarchia eidetica: la componente dell'estensione.

\begin{abstract}
Allo spazio ideale spetta per noi un a-priori universale e sistematicamente unitario, una teoria sistematica unitaria infinita, la quale, nonostante la sua infinità, è in sé conclusa e, partendo da concetti o da proposizioni di tipo assiomatico, permette di costruire in un'univocità deduttiva qualsiasi forma delimitabile nello spazio. Tutto ciò che esiste 'idealiter' nello spazio geometrico, è preliminarmente e univocamente definito in tutte le sue determinazioni. Il nostro pensiero apodittico 'scopre' soltanto, mediante concetti, proposizioni, conclusioni, dimostrazioni, procedendo gradualmente all'infinito, ciò che è già in sé e preliminarmente vero (HUSSERL, 1961, p.54).
\end{abstract}

I plena vivono per loro stessa natura la dimensione del pressappoco, dell'inesattezza e della singolarità. Essi non sono, in altri termini, approssimazioni verso alcun ideale, quindi non sono entità perfettamente e univocamente deducibili. Il fatto che la tipicità e la gradualità della dimensione propriamente qualitativa e contenutistica dell'ontologia renda non idealizzabile quella ontologia stessa (non esiste un rosso ideale così come non esiste un suono ideale, mentre è possibile concepire un triangolo ideale), fa sì che la matematizzazione diretta dei plena risulti di fatto impossibile. Solo l'estensione ha, quindi, una forma-limite, non un plenum. Solo nel caso dell'estensione, infatti, la gradualità può andare nella direzione di una maggiore o minore perfezione ed è proprio il limite verso cui tende questa maggiore o minore perfezione che chiamiamo idealità.

L'impossibilità di matematizzare direttamente i plena era nota anche a Galileo, che non a caso operava una espulsione delle qualità secondarie dall'assetto ontologico del mondo. Solo le qualità primarie (moto, estensione) risultano infatti matematizzabili. Per poter operare una matematizzazione delle datità sensibili sembra quindi rendersi necessario "svuotare" l'ontologia materiale da ogni contenuto, in modo da ottenere entità dotate di quella esattezza e univoca

\footnotetext{
${ }^{24} \mathrm{~A}$ considerazioni di questo tipo è legato il problema della matematizzazione, diretta e indiretta, dei plena, cioè delle datità sensibili, quelle datità che costituiscono il contenuto specifico degli atti intuitivi. Si vedano, a tale proposito, anche le (HUSSERL, 1961,p. 63 e ss).
} 


\section{Aoristo)))))}

\section{International Journal of Phenomenology, Hermeneutics and Metaphysics}

determinabilità di cui la datità originaria è priva. Espressione di questa univoca determinabilità è la misurazione.

Mediante il concetto di misurazione la fisica matematica coglie e seleziona quegli aspetti degli oggetti intuitivi che interessano la matematica in quanto matematica delle forme (le quali ineriscono in modo essenziale alla nozione di cosa, costituendone tuttavia solo un aspetto), nel tentativo di giungere a una conoscenza oggettiva della entità concreta nella sua globalità.

Esattamente in questa operazione - quella della matematizzazione indiretta dei plena - consiste la "sorprendente ipotesi galileaiana". La cosa fisica, composta da estensione e plena, viene per così dire "scorporata". Il passo successivo consiste nel considerare ogni mutamento complessivo del plenum come avente una propria controfigura nella sfera delle forme, controfigura che risulta infine interpretata come avente un nesso causale necessario con la prima ${ }^{25}$.

Da qui l'idea che tutto ciò che si manifesta con qualità sensibili debba avere un indice matematico corrispettivo agli eventi idealizzati. Questa idea, che sta alla base della matematizzazione indiretta dei plena, costituisce un processo in grado di determinare obiettivamente tutti gli eventi. Con la idealizzazione del mondo proposta dall'ipotesi galileiana viene così affermata l'ovvietà di una causalità esatta universale che sostituisca quella concreta. La chiusura del cerchio è costituita dall'idea di previsione relativa alla sfera delle intuizioni immediate.

Il punto, tuttavia, è che tale operazione finisce per escludere parte integrante del dato originario: i plena, appunto. La natura, in definitiva, non è costruttivamente determinabile in tutti i suoi aspetti ma solo in alcuni aspetti opportunamente selezionati. In questo consiste lo "[...] svuotamento di senso"operato dalla matematizzazione della natura a opera di Galileo e per questo Galileo può essere interpretato come "un genio che scopre e insieme occulta"(HUSSERL, 1976, p.81).

Nella matematizzazione geometrica e scientifico-naturale, noi commisuriamo così al mondo-della-vita - al mondo che ci è costantemente e realmente dato nella nostra vita concreta che si svolge in esso -, nella aperta infinità di un'esperienza possibile, un ben confezionato abito ideale, quello delle cosiddette verità obiettivamente scientifiche; costruiamo cioè (o almeno speriamo di costruire), attraverso un metodo realmente praticabile in tutti i particolari e costantemente verificato, determinate induzioni numeriche per i plena sensibili reali e possibili delle forme concrete-intuitive del mondo-della-vita; e proprio così attingiamo la possibilità di una previsione degli accadimenti concreti del mondo, di quegli eventi che non sono più o non sono ancora realmente dati, degli eventi, cioè, intuitivi del mondodella-vita, attingiamo cioè la possibilità di una previsione che supera infinitamente la portata della previsione quotidiana.

L'abito ideale che si chiama "[...] matematica e scienza naturale matematica" oppure l'ambito simbolico delle teorie simbolico-matematiche, abbraccia,

\footnotetext{
${ }^{25}$ Si potrebbe sostenere quindi che l'originario rapporto di fondazione fra forma e plenum venga, mediante l'ipotesi galileiana, convertito in un rapporto di causazione necessario del tutto artificiale.
} 


\section{Aoristo)))))}

International Journal of Phenomenology, Hermeneutics and Metaphysics

riveste tutto ciò che per gli scienziati e per le persone colte, in quanto "natura obiettivamente reale e vera" rappresenta il mondo-della-vita. L'abito ideale fa sì che noi prendiamo per il vero essere quello che invece è soltanto un metodo, un metodo che deve servire a migliorare mediante "previsioni scientifiche"in un "progressus in infinitum", le previsioni grezze, le uniche possibili nell'ambito di ciò che è realmente esperito ed esperibile nel mondo-della-vita; l'abito ideale poté far sì che il senso proprio del metodo, delle formule, delle "teorie" rimanesse incomprensibile e che durante l'elaborazione ingenua del metodo non venisse mai compreso (HUSSERL, 1976, p.8081).

\section{MOTIVAZIONE E ASSURDITÀ EFFETTIVA}

Abbiamo visto come il rapporto di fondazione fra momenti o parti non indipendenti risulti essere: necessario affinché si possa parlare di dato; non "estraibile" in un autonomo principio unificatore; contenutistico, cioè fondato sulla specifica natura delle determinazioni materiali coinvolte.

La violazione del rapporto di fondazione porta, nella prospettiva fenomenologica, al contro senso (Widersinn) materiale. L'impossibilità materiale determinata dalla violazione del rapporto di fondazione deve essere concettualmente distinta dalla impossibilità determinata dalla insensatezza o non-senso (Unsinning). Quest'ultima si verifica quando la struttura della noesi non risulta formalmente ben formata come nel caso "verde è e" (HUSSERL, 1984, p.319-320). L'impossibilità determinata dal non-senso concerne, possiamo dire, l'aspetto sintattico della noesi: in essa l'atto semplicemente non intende "qualcosa", quindi è privo di un orientamento intenzionale. Al contrario, l'impossibilità sancita dal contro-senso concerne l'aspetto semantico della noesi: in essa, infatti, l'atto risulta diretto verso oggetti impossibili a priori, perché i momenti che ne costituiscono il senso si pongono "l'uno contro l'altro". L'intenzione ha, in questo caso, una struttura sintatticamente ben formata e tuttavia l'oggetto al quale essa è rivolta non è obiettivamente unificabile.

Sembra tuttavia lecito proporre un'ulteriore distinzione fra contro-senso materiale (un colore senza estensione; un carattere d'atto, come la percezione, non connesso a uno specifico contenuto; un timbro senza altezza) e contro-senso formale come nel caso del quadrato rotondo. Quest'ultimo risulta infatti impossibile perché contravviene alla legge essenziale stando alla quale due specie dello stesso genere non possono convivere all'interno della stessa singolarità. Un'interpretazione di questo tipo sembra suggerita dallo stesso Husserl:

Le singolarità eidetiche contenute disgiuntamente in un concreto sono necessariamente "eterogenee" con riguardo alla legge ontologicoformale secondo cui due singolarità eidetiche del medesimo genere non possono essere unite in una sola essenza o, come pure si dice: le differenze ultime di un genere sono "incompatibili" tra loro. Quindi ogni singolarità, inserita in un concreto, considerata come differenza, 


\section{Aoristo)))))}

\section{International Journal of Phenomenology, Hermeneutics and Metaphysics}

conduce a un sistema separato di specie e di generi (HUSSER, 1976, p. 38).

Queste distinzioni si appiattiscono in un'unica dimensione nella critica che Moritz Schlick rivolge alla nozione di a priori materiale, in cui esempi come "ogni nota ha un'altezza e un'intensità", "una stessa superficie non può essere al tempo stesso verde e rossa" e "una superficie è rossa e non è rossa" vengono considerati analoghi (SCHLICK, 1987), mentre in una prospettiva fenomenologica rimandano a logiche di tipo differente: contro-senso materiale nel primo esempio, contro-senso formale nel secondo e non senso formale nel terzo.

I due tipi di impossibilità, non senso e contro-senso (sia esso materiale o formale), risultano a loro volta distinti da un terzo tipo di impossibilità, quello che fa esplicito riferimento alla costituzione dell'oggetto noematico o trascendenza fenomenologica. In quest'ultimo caso ciò che è infatti in gioco non è né la sensatezza dell'oggetto (la quale rimanda alla correttezza formale della noesi), né l'identificazione intuitiva di una determinazione dell'oggetto (la quale rimanda all'ideazione), e nemmeno la reciproca fondazione di determinazioni dell'oggetto (la quale nella fenomenologia rimanda a una necessaria legalità materiale). Ciò che è in gioco è, infatti, la sintesi delle diverse apparizioni della cosa, che a loro volta sono il risultato dei rapporti di fondazione fra le parti non indipendenti dell'oggetto.

La nozione di sintesi noematica, così come viene presentata da Husserl in Ideen I, comporta l'assunzione di un polo unitario, di una $X$ determinata e determinabile intorno alla quale ruotano tutte le apparizioni dell'oggetto, polo che non deve tuttavia essere in alcun modo confuso con un substrato di tipo sostanziale. Non ruotando i predicati e le determinazioni della cosa intorno ad alcun "perno"sostantivo, la X determinabile a cui Husserl fa riferimento può essere facilmente ricondotta, ed è stata ricondotta di fatto dallo stesso autore in varie occasioni, alla nozione kantiana di idea.

Caratteristica dell'ideazione che intuisce un'"idea" kantiana, caratteristica che pertanto non ne diminuisce l'evidenza, è appunto che l'adeguata determinazione del suo contenuto $[\ldots]$ sia irraggiungibile. Ci sono oggetti - e rientrano in questi tutti gli oggetti trascendenti, tutte le "realtà naturali" comprese dal titolo natura e mondo - che non possono essere dati in piena determinatezza e allo stesso modo in piena intuitività in nessuna coscienza conclusa. Tuttavia come "idea" (in senso kantiano) la perfetta datità è predelineata; questa idea designa un sistema, assolutamente determinato nel suo tipo eidetico, all'interno del quale hanno luogo processi fenomenici infiniti, oppure un campo di questi processi, un continuum fenomenico determinato a priori con dimensioni diverse ma determinate, e governato da una rigida legalità eidetica. [...] Se un'unità conclusa di questo decorso, e quindi un atto finito, sia pur mobile, è inconcepibile a causa della onnilaterale infinità del continuum (altrimenti avremo 


\section{Aoristo)))))}

International Journal of Phenomenology, Hermeneutics and Metaphysics

un'assurda infinità finita), tuttavia l'idea di questo continuum e l'idea della perfetta datità come è da esso prefigurata sono idee evidenti così evidenti come appunto può esserlo un'"idea" in senso kantiano che designa attraverso la sua scienza il suo proprio tipo di evidenza (HUSSERL, 1976, p. 355).

L'oggetto, definito come punto di annodamento, portatore dei predicati espressi dal Sinn noematico, punto centrale di unità intorno al quale ruotano le indefinite determinazioni dell'oggetto secondo una "rigida legalità eidetica", "l'oggetto immodificato, l'identico di tutti i modi di apparizione, l'identico di tutti gli orientamenti" sembra costituire, da tale punto di vista, una unità logicamente prioritaria rispetto a ogni complesso determinato di aspetti dell'oggetto stesso. Ed è, inoltre, proprio tale priorità logica a costituire il carattere determinato e pre-delineato dell'oggetto a cui Husserl fa esplicito riferimento. L'oggetto fenomenologico, stando a tale prospettiva, sembra quindi caratterizzato da un lato da apertura e incompletezza (esso è essenzialmente inadeguato proprio perché in grado di accogliere sempre successive determinazioni) e, dall'altro, da quel carattere determinato e pre-definito che legittima l'uso del termine "regola". "Ma è una generale intuizione essenziale che ogni datità imperfetta (ogni noema che si offre inadeguatamente) racchiude in sé una regola che governa la possibilità ideale del suo perfezionamento" (HUSSERL, 1976, p. 369).

Se andiamo tuttavia a indagare che cosa intenda Husserl per "regola", troveremo tuttavia alcuni aspetti rilevanti di tale nozione che si allontanano da quel sistema predeterminato e pre-delineato che sembra a un primo sguardo regolare l'unità, la sintesi delle apparizioni di un oggetto ridimensionando così fortemente quella analogia fra sintesi noematica e idea kantiana che lo stesso Husserl esplicitamente suggerisce.

L'uso del termine "regola" sembra infatti escludere non solo un substrato o comunque un perno di tipo sostanziale al quale le apparizioni della cosa si possono riferire, ma anche un principio unificatore che risulti autonomo e indipendente rispetto alle apparizioni. Ancora una volta, come già nel caso della relazione di fondazione, ciò che è assente è l'idea della "estraibilità" di un qualche momento di unità.

L'elemento che permette di racchiudere, sia pure in modo indefinitamente aperto, tutti i possibili e coerenti modi di darsi di un oggetto è il legame motivazionale che sussiste fra $\mathrm{i}$ fenomeni. L'oggetto inteso nel suo complesso risulta infatti interpretato come sintesi, o polo unitario, di tutte le possibili determinazioni motivate da un'esperienza attuale. Ed è proprio l'introduzione della nozione di legame motivazionale a ridimensionare drasticamente l'analogia fra " $X$ determinabile" e "idea kantiana". La sintesi unitaria di tutte le possibili apparizioni ma anche determinazioni concettuali dell'oggetto, a cui Husserl fa riferimento, non è infatti una struttura né formale né ideale, bensì una struttura sostanzialmente condizionata da elementi contenutistici e "attuali". Un oggetto sarà identificato con lo stesso oggetto se istituisce un legame motivazionale con un'apparenza iniziale, originaria: in questo senso, è ciò che attualmente si dà a motivare le ulteriori 


\section{Aoristo)))))}

\section{International Journal of Phenomenology, Hermeneutics and Metaphysics}

apparizioni della cosa, da quelle sensibili (il lato non visto della cosa) ${ }^{26}$ fino a quelle più astratte e concettuali.

Che sussista un determinato legame motivazionale non è necessario: la sintesi delle apparizioni, come Husserl riconosce apertamente, può rompersi e quel determinato corso d'esperienza $\mathrm{o}$, il che è lo stesso, quel determinato legame motivazionale, disintegrarsi. Ma che esista un certo legame motivazionale fra i fenomeni, questo è assolutamente necessario affinché si possa parlare propriamente di esperienza. Tale nozione richiede infatti una qualche sintesi o integrazione fra le apparenze.

Il poter essere esperita non allude a una vuota possibilità logica, ma a una possibilità motivata dalla connessione dell'esperienza. Questa è un'intera concatenazione di motivazioni che integra continuamente in sé nuove motivazioni e trasforma quelle già formate. (HUSSERL, 1976, 117).

La necessità, ai fini della determinazione di un oggetto e della sintesi delle sue apparenze, di istituire un legame genetico-motivazionale con una esperienza effettiva originaria, esclude la possibilità della cosa in sé, della trascendenza assoluta o metafisica. Quest'ultima, infatti, comporta una "[...] assolutizzazione filosofica del mondo, che è del tutto estranea alla considerazione naturale del mondo stesso" (HUSSERL, 1961, p. 140), assolutizzazione che, pur non identificandosi né con il non senso né con la violazione dei rapporti di fondazione fra parti non indipendenti dell'oggetto, costituisce nondimeno una chiara assurdità fenomenologica ${ }^{27}$.

Dal punto di vista 'logico', l'ipotesi di una realtà fuori di questo mondo è certo possibile, in quanto essa non implica una contraddizione formale. Ma se ci interroghiamo sulle condizioni essenziali della sua validità, se ci interroghiamo sul tipo di legittimazione richiesta dal suo senso [...] dovremo riconoscere che una simile realtà deve necessariamente poter essere esperita, e non soltanto da parte di un soggetto concepito come una vuota possibilità logica, ma da parte di un io; questa realtà deve poter essere esperita come un'unità che può essere esibita nelle connessioni d'esperienza di questo io [...]. Riflettendo su tutto questo ci si persuade che la possibilità logico-formale di altre realtà fuori del mondo, fuori dell'unico mondo spazio-temporale, che è fissato dalla nostra esperienza attuale, è concretamente una assurdità (HUSSERL, 1961, p.118-119).

\footnotetext{
26 “Le cose 'viste' sono già sempre qualcosa di più di ciò che di esse 'realmente e propriamente' vediamo. Vedere, percepire, significa per essenza avere-in-persona” e insieme progettare (vorhaben), pre-sumere (vormeinen). Qualsiasi prassi, con tutti i suoi progetti, implica induzioni" (HUSSERL, 1961, p. 80).

${ }^{27}$ Ho tentato di distinguere controsenso materiale e assurdità effettiva in Lanfredini (2004), p. 155-173.
} 


\section{Aoristo)))))}

\section{International Journal of Phenomenology, Hermeneutics and Metaphysics}

Si noti, tuttavia, come la negazione di una trascendenza assoluta non comporti la negazione di una trascendenza fenomenologica tout court e nemmeno la negazione di entità inosservabili. Al contrario, Husserl è perfettamente consapevole del fatto che la determinazione teoretica delle cose sensibilmente esperite richieda uno "svuotamento" delle qualità sensibili stesse a favore di qualità non più sensibili o formali. Tale procedimento di formalizzazione è del tutto legittimo a condizione, tuttavia, che non venga ripristinata la "mitologia" della cosa in assoluto separata, causa oggettiva di manifestazioni sensibili.

Un essere trascendente è dato tramite certe connessioni dell'esperienza. Dato direttamente e con crescente perfezione all'interno di un flusso continuo di percezioni che si rivelano concordanti, attraverso un pensiero che, a partire dall'esperienza, elabora determinate forme metodiche, l'essere trascendente perviene più $\mathrm{o}$ meno mediatamente $\mathrm{a}$ una determinazione teoretica evidente $\mathrm{e}$ progressiva. [...] La cosa dei fisici non è estranea a ciò che si manifesta sensibilmente in carne ed ossa; essa si annuncia invece in ciò che si manifesta e (per insopprimibili motivi aprioristici) si annuncia originalmente soltanto in ciò che si manifesta (HUSSERL, 1961, p. 125-1312).

Con ciò Husserl sembra confermare ancora una volta un empirismo radicale e sostanzialmente genetico.

Bisogna qui tenere presente che ciò che le cose sono - le uniche cose su cui noi possiamo pronunciare giudizi, sul cui essere e non essere, sull'essere così o sull'essere diversamente noi possiamo disputare o decidere razionalmente - lo sono in quanto cose dell'esperienza. L'esperienza sola è quella che fissa il loro senso e, poiché si tratta di cose di fatto, sarà l'esperienza attuale con le sue determinazioni e ordinate connessioni d'esperienza che fisserà il loro senso (HUSSERL, 1961, p. 116).

\section{CONCLUSIONE}

L'analisi fenomenologica dell'oggetto è orientata non tanto a indagare le "condizioni di possibilità" dell'oggetto quanto ad analizzare ciò che è "effettivamente contenuto" nel dato, cioè in ciò che primariamente e evidentemente si manifesta. In questo senso sembra plausibile scorgere nella fenomenologia husserliana un'impostazione sostanzialmente empirista.

In questa direzione possono essere lette tutte le principali nozioni nelle quali la prospettiva fenomenologia si incardina: la priorità genetica dell'atto intuitivo e più specificamente percettivo; la nozione di trascendenza fenomenologia analiticamente distinta dalla trascendenza assoluta o metafisica; il carattere imprescindibile del concetto di motivazione e la caratterizzazione fenomenologia della sintesi unitaria delle apparenze

\footnotetext{
28 In questo senso sembra quindi lecito contrapporre il nesso genetico-motivazionale al nesso causale: "[...] è quindi contraddittorio connettere causalmente le cose dei sensi e quelle della fisica" (HUSSERL, 1961, p. 132).
} 


\section{Aoristo)))))}

International Journal of Phenomenology, Hermeneutics and Metaphysics

dell'oggetto; il processo ideativo nella misura in cui si contrappone non solo alla teoria della astrazione empirista ma anche alla teoria strutturale e formale del concetto; la concezione delle essenze fenomenologiche come essenze inesatte, fluenti, morfologiche, strutturalmente non matematizzabili.

Infine, anche la nozione che questo lavoro aveva l'intento di mettere a fuoco, la nozione cioè di a priori materiale, sembra contribuire a sostenere l'immagine radicalmente empirista della fenomenologia husserliana. Tale nozione, tecnicamente intesa come nesso fondazionale che vige fra momenti o parti non indipendenti dell'intero intuitivo rimanda infatti a un'analisi tutta interna alla nozione primitiva di dato, un'analisi cioè che ha la pretesa generale (sulla quale è tuttavia possibile dissentire) di analizzare la struttura dell'esperienza in sé stessa, senza ricorso immediato a categorie o a principi teorici.

\section{REFERENZE}

CASSIRER, E. Sostanza e funzione. Firenze: La Nuova Italia, 1973.

FRIEDMAN, M. Parting of the ways - Carnap, Cassirer, and Heidegger. Carus: Publishing Company, 2004.

HUSSERL, E. Die Idee der Phänomenologie. The Hague: Martinus Nijoff, 1950.

. Die Krisis der europäischen Wissenschaften und die transzendentale Phänomenologie. Den

Haag: Martinus Nijoff, 1959 (trad. it. Crisi delle scienze europee. Milano: Mondatori, 1961).

. Ideen zu einer reinen Phänomenologie und phänomenologische Philosophie. Erstes Buch:

Allgemeine Einführung in die reine Phänomenologieln: Husserliana III/1. Den Haag: Martinus Nijoff, 1976; (trad. it. Idee per una fenomenologia pura e per una filosofia fenomenologica, vol. I. Torino: Einaudi, 2002).

. Logische Untersuchungen, Zweiter Teil/1: Untersuchungen zur Phänomenologie und Theorie

der Erkenntnis. Zweiter Teil/2: Elemente einer Phänomenologische Aufklärung der Erkenntnis, Husserliana XIX/1-2. Den Haag: Martinus Nijoff, 1984 (trad. it. Ricerche logiche, 2 v., Il Saggiatore, Milano, 1968).

LANFREDINI, R. Fenomeno e cosa in sé: Tre livelli di impossibilità fenomenologica. In: Fenomenologia applicata. (Ed.) R. Lanfredini. Milano: Guerini, 2004.

. L' ontologia fenomenologica. In: Oltrecorrente. 9, ott. 2004, p. 135-144

La fenomenologia come scienza di essenze inesatti. In: Rivista di estetica, n. 22, v.1, (2003), p.

100-108.

Oggetti e paradigmi. Per una concezione interattiva della conoscenza scientifica. Roma:

Theoria, 1988.

PARRINI, P. Conoscenza e realtà. Bari: Laterza, 1995.

. L'empirismo logico. Aspetti storici e prospettive teoriche. Milano: Carocci, 2002.

POPPER, K. The logic of scientific discovery. London

SCHLICK, M. Esiste un a priori materiale. In: Forma e contenuto. Torino: Boringhieri, 1987.

SEARLE, J. Intentionality. An Essay in the Philosophy of Mind. Cambridge: Cambridge University Press, 1983(

Inserito: 4 giugno 2021

Accettato: 1 luglio 2021

Roberta Lanfredini

Toledo, v. 4, n*2(2021) p. 98-128 